\title{
Well-posedness for 3D nematic liquid crystal flows with damping
}

\author{
Hui Liu ${ }^{1 *}$, Chengfeng Sun ${ }^{2}$ and Jie Xin ${ }^{1,3}$
}

\section{"Correspondence:}

liuhuinanshi@qfnu.edu.cn

'School of Mathematical Sciences,

Qufu Normal University, Qufu,

P.R. China

Full list of author information is

available at the end of the article

\section{Springer}

\begin{abstract}
The three-dimensional nematic liquid crystal flows with damping are considered in this paper. The existence and uniqueness of strong solutions for the 3D nematic liquid crystal flows with damping are proved for $\beta \geq 4$ with any $\alpha>0$.
\end{abstract}

Keywords: Liquid crystal flows; Well-posedness; Strong solution

\section{Introduction}

In this paper, we consider the following three-dimensional nematic liquid crystal flows with damping:

$$
\left\{\begin{array}{l}
\partial_{t} u-v \Delta u+(u \cdot \nabla) u+\alpha|u|^{\beta-1} u+\nabla p=-\lambda \nabla \cdot(\nabla d \odot \nabla d), \\
\partial_{t} d+(u \cdot \nabla) d=\gamma(\Delta d-f(d)), \\
\nabla \cdot u=0 \\
\left.u\right|_{\partial D}=\left.d\right|_{\partial D}=0, \\
u(x, 0)=u_{0}(x), \quad d(x, 0)=d_{0}(x) .
\end{array}\right.
$$

Here, $x \in D \subseteq \mathbb{R}^{3}$ is a bounded domain with the boundary $\partial D$ and $t>0 . u=u(x, t)$ is the velocity field of the flow, $d=d(x, t)$ represents the (averaged) macroscopic/continuum molecule orientation and $p$ is the pressure. $v, \lambda, \gamma, \alpha$ are positive constants, $\beta \geq 1$ and $f(d)=\frac{1}{\eta^{2}}\left(|d|^{2}-1\right) d(\eta>0)$. The $3 \times 3$ matrix is given by $(\nabla d \odot \nabla d)_{i j}=\partial_{i} d \cdot \partial_{j} d$ for $(1 \leq$ $i, j \leq 3)$. For simplicity, we set $v=\gamma=\lambda=\eta=1$.

Recently, the 3D nematic liquid crystal flows were proposed by Lin $([1,2])$ and have been extensively investigated. The damping term describes many physical situations such as drag or friction effects, porous media flow, some dissipative mechanisms. When $d=0$, the problem (1) reduces to the three-dimensional Navier-Stokes equations with damping. In [3-6], the well-posedness of the three-dimensional Navier-Stokes equations with damping is proved for $\beta>3$ with any $\alpha>0$ and $\alpha \geq \frac{1}{4}$ as $\beta=3$. The global existence of weak solutions of the 3D nematic liquid crystal flow was proved in [7]. In [8], the existence and uniqueness of strong solutions for the 3D magneto-micropolar equations were proved for $\beta \geq 4$ with any $\alpha>0$.

(c) The Author(s) 2020. This article is licensed under a Creative Commons Attribution 4.0 International License, which permits use, sharing, adaptation, distribution and reproduction in any medium or format, as long as you give appropriate credit to the original author(s) and the source, provide a link to the Creative Commons licence, and indicate if changes were made. The images or other third party material in this article are included in the article's Creative Commons licence, unless indicated otherwise in a credit line to the material. If material is not included in the article's Creative Commons licence and your intended use is not permitted by statutory regulation or exceeds the permitted use, you will need to obtain permission directly from the copyright holder. To view a copy of this licence, visit http://creativecommons.org/licenses/by/4.0/. 
This paper is organized as follows. In Sect. 2, we will prove the existence and uniqueness of strong solutions for the 3D nematic liquid crystal flows with damping for $\beta \geq 4$ with any $\alpha>0$. Moreover, we get the following main result.

Theorem 1.1 Let $\left(u_{0}, d_{0}\right) \in H^{1}(D) \times H^{2}(D)$ such that $\nabla \cdot u_{0}=0$, for $\beta \geq 4$ with any $\alpha>0$. Then the problem (1) has a unique strong solution $(u, d)$ satisfying for any given $T>0$

$$
\begin{aligned}
& u \in L^{\infty}\left(0, T ; H^{1}\right) \cap L^{2}\left(0, T ; H^{2}\right) \cap L^{\beta+1}\left(0, T ; L^{\beta+1}(D)\right), \\
& d \in L^{\infty}\left(0, T ; H^{1}\right) \cap L^{\infty}\left(0, T ; H^{2}\right) \cap L^{2}\left(0, T ; H^{3}\right), \\
& |u|^{\frac{\beta-1}{2}} \nabla u \in L^{2}\left(0, T ; L^{2}(D)\right), \quad \nabla|u|^{\frac{\beta+1}{2}} \in L^{2}\left(0, T ; L^{2}(D)\right) .
\end{aligned}
$$

\section{Proof of Theorem 1.1}

In this section, $C$ represents a nonnegative constant whose value may be different from line to line. Multiplying the first equation of Eq. (1) by $u$ and the second equation of (1) by $-\Delta d+f(d)$, integrating the result over $D$, and summing their results, then we have

$$
\frac{1}{2} \frac{d}{d t} \int_{D}\left(|u|^{2}+|\nabla d|^{2}+2 F(d)\right) d x+\int_{D}\left(|\nabla u|^{2}+\alpha|u|^{\beta+1}+|\Delta d-f(d)|^{2}\right) d x=0
$$

here $f(d)=\nabla F(d),((u \cdot \nabla) u, u)=(u, \nabla p)=((u \cdot \nabla) d, f(d))=\left(u, \nabla \frac{|\nabla d|^{2}}{2}\right)=0$ and $\nabla \cdot(\nabla d \odot$ $\nabla d)=\nabla\left(\frac{|\nabla d|^{2}}{2}\right)+\Delta d \cdot \nabla d$, i.e., $F(d)=\frac{|d|^{4}}{4}-\frac{|d|^{2}}{2}$. Then it is easy to get

$$
\|u\|_{L^{\infty}\left(0, T ; L^{2}\right)}+\|u\|_{L^{\beta+1}\left(0, T ; L^{\beta+1}\right)}+\|u\|_{L^{2}\left(0, T ; H^{1}\right)} \leq C .
$$

Multiplying the second equation of (1) by $|d|^{2} d$, it is easy to get

$$
\frac{1}{4} \frac{d}{d t} \int_{D}|d|^{4} d x+\frac{1}{2}\left\|\nabla|d|^{2}\right\|_{L^{2}}^{2}+\int_{D}\left(|d|^{2}|\nabla d|^{2}+|d|^{6}\right) d x=\int_{D}|d|^{4} d x .
$$

Applying the Gronwall inequality, then we have

$$
\|d(t)\|_{L^{4}}^{4}+\int_{0}^{t}\left\|\nabla|d|^{2}\right\|_{L^{2}}^{2} d s+\int_{0}^{t} \int_{D}\left(|d|^{2}|\nabla d|^{2}+|d|^{6}\right) d x d s \leq C\left(t, d_{0}\right) .
$$

Multiplying the second equation of (1) by $f(d)$, we deduce

$$
\frac{d}{d t} \int_{D} F(d) d x=\int_{D}\left(\Delta d f(d)-|f(d)|^{2}\right) d x .
$$

Adding (2)-(6) and using the Gronwall inequality and $f(d)=\left(|d|^{2}-1\right) d$, we have

$$
\|d\|_{L^{\infty}\left(0, T ; H^{1}\right)}+\|d\|_{L^{2}\left(0, T ; H^{2}\right)} \leq C .
$$

Multiplying the first equation of (1) by $-\Delta u$, it is easy to get

$$
\begin{aligned}
& \frac{1}{2} \frac{d}{d t}\|\nabla u\|_{L^{2}}^{2}+\|\Delta u\|_{L^{2}}^{2}+\alpha\left\||u|^{\frac{\beta-1}{2}} \nabla u\right\|_{L^{2}}^{2}+\frac{4 \alpha(\beta-1)}{(\beta+1)^{2}}\left\|\nabla|u|^{\frac{\beta+1}{2}}\right\|_{L^{2}}^{2} \\
& \quad=\int_{D}(u \cdot \nabla) u \cdot \Delta u d x+\int_{D} \nabla d \Delta d \Delta u d x .
\end{aligned}
$$


Taking $\Delta$ on the second equation of (1) and dotting with $\Delta d$, we get

$$
\begin{aligned}
\frac{1}{2} \frac{d}{d t}\|\Delta d\|_{L^{2}}^{2}+\|\nabla \Delta d\|_{L^{2}}^{2}= & -\int_{D} \Delta f(d) \Delta d d x-\int_{D} \Delta u \nabla d \Delta d d x \\
& -2 \sum_{i, k=1}^{3} \int_{D} \nabla u_{i} \partial_{i} \nabla d_{k} \Delta d_{k} d x
\end{aligned}
$$

Adding (8) and (9), we have

$$
\begin{aligned}
\frac{1}{2} & \frac{d}{d t}\left(\|\nabla u\|_{L^{2}}^{2}+\|\Delta d\|_{L^{2}}^{2}\right)+\|\Delta u\|_{L^{2}}^{2}+\|\nabla \Delta d\|_{L^{2}}^{2}+\alpha\left\||u|^{\frac{\beta-1}{2}} \nabla u\right\|_{L^{2}}^{2} \\
& +\frac{4 \alpha(\beta-1)}{(\beta+1)^{2}}\left\|\nabla|u|^{\frac{\beta+1}{2}}\right\|_{L^{2}}^{2} \\
= & \int_{D}(u \cdot \nabla) u \cdot \Delta u d x-\int_{D} \Delta f(d) \Delta d d x-2 \sum_{i, k=1}^{3} \int_{D} \nabla u_{i} \partial_{i} \nabla d_{k} \Delta d_{k} d x \\
= & \sum_{i=1}^{3} I_{i}(t) .
\end{aligned}
$$

For $I_{1}(t)$, using the Young inequality and the Hölder inequality, it is easy to get, for any $\beta>3$,

$$
\begin{aligned}
\left|I_{1}(t)\right| & \leq \frac{1}{4}\|\Delta u\|_{L^{2}}^{2}+C \int_{D}|u|^{2}|\nabla u|^{\frac{4}{\beta-1}}|\nabla u|^{2-\frac{4}{\beta-1}} d x \\
& \leq \frac{1}{4}\|\Delta u\|_{L^{2}}^{2}+\frac{\alpha}{2} \int_{D}|u|^{\beta-1}|\nabla u|^{2} d x+C\|\nabla u\|_{L^{2}}^{2} .
\end{aligned}
$$

Inspired by [3-5] and exists $\theta>0$, we get $1-\frac{1}{2 \theta} \geq 0$ and $\alpha-\frac{\theta}{2} \geq 0$. Then we get the above estimate easily for $\alpha \geq \frac{1}{4}$ as $\beta=3$.

For $I_{2}(t)$, integrating by parts, applying the Hölder inequality and the Young inequality, we get

$$
\begin{aligned}
I_{2}(t) & =\sum_{i=1}^{3} \int_{D} \partial_{i}\left(|d|^{2} d\right) \partial_{i} \Delta d d x-\sum_{i=1}^{3} \int_{D} \partial_{i} d \partial_{i} \Delta d d x \\
& \leq C\left(\|\nabla d\|_{L^{6}}\|\nabla \Delta d\|_{L^{2}}\|d\|_{L^{6}}^{2}+\|\nabla d\|_{L^{2}}\|\nabla \Delta d\|_{L^{2}}\right) \\
& \leq \frac{1}{4}\|\nabla \Delta d\|_{L^{2}}^{2}+C\left(\|\nabla d\|_{L^{2}}^{2}+\|\nabla d\|_{L^{2}}^{4}\|\Delta d\|_{L^{2}}^{2}\right) \\
& \leq \frac{1}{4}\|\nabla \Delta d\|_{L^{2}}^{2}+C\|\Delta d\|_{L^{2}}^{2}+C .
\end{aligned}
$$

For $I_{3}(t)$, since $\frac{2}{\beta-2} \leq 1$ for $\beta \geq 4$, by using the Hölder, Gagliardo-Nirenberg and Young inequalities, we get

$$
\begin{aligned}
I_{3}(t) & =2 \sum_{i=1}^{3}\left(\int_{D} u_{i} \partial_{i} \nabla d \nabla \Delta d d x+\int_{D} u_{i} \partial_{i} \nabla^{2} d \Delta d d x\right) \\
& \leq C\|u\|_{L^{\beta+1}}\|\Delta d\|_{L^{\frac{2(\beta+1)}{\beta-1}}}\|\nabla \Delta d\|_{L^{2}}
\end{aligned}
$$




$$
\begin{aligned}
& \leq C\|u\|_{L^{\beta+1}}\|\Delta d\|_{L^{2}}^{\frac{\beta-2}{\beta+1}}\|\nabla \Delta d\|_{L^{2}}^{\frac{\beta+4}{\beta+1}} \\
& \leq \frac{1}{4}\|\nabla \Delta d\|_{L^{2}}^{2}+C\|u\|_{L^{\beta+1}}^{\frac{2(\beta+1)}{\beta-2}}\|\Delta d\|_{L^{2}}^{2} \\
& \leq \frac{1}{4}\|\nabla \Delta d\|_{L^{2}}^{2}+C\left(1+\|u\|_{L^{\beta+1}}^{\beta+1}\right)\|\Delta d\|_{L^{2}}^{2} .
\end{aligned}
$$

Adding (10)-(13), it is easy to get

$$
\begin{aligned}
& \frac{d}{d t}\left(\|\nabla u\|_{L^{2}}^{2}+\|\Delta d\|_{L^{2}}^{2}\right)+\|\Delta u\|_{L^{2}}^{2}+\|\nabla \Delta d\|_{L^{2}}^{2}+\left\||u|^{\frac{\beta-1}{2}} \nabla u\right\|_{L^{2}}^{2}+\left\|\nabla|u|^{\frac{\beta+1}{2}}\right\|_{L^{2}}^{2} \\
& \quad \leq C\left(1+\|u\|_{L^{\beta+1}}^{\beta+1}\right)\left(\|\Delta d\|_{L^{2}}^{2}+\|\nabla u\|_{L^{2}}^{2}\right)+C .
\end{aligned}
$$

Applying the Gronwall inequality and (3), we have

$$
\begin{aligned}
& \|\nabla u(t)\|_{L^{2}}^{2}+\|\Delta d(t)\|_{L^{2}}^{2} \\
& \quad+\int_{0}^{t}\left(\|\Delta u\|_{L^{2}}^{2}+\|\nabla \Delta d\|_{L^{2}}^{2}+\left\||u|^{\frac{\beta-1}{2}} \nabla u\right\|_{L^{2}}^{2}+\left\|\nabla|u|^{\frac{\beta+1}{2}}\right\|_{L^{2}}^{2}\right) d s \\
& \quad \leq C\left(t, u_{0}, d_{0}\right) .
\end{aligned}
$$

Next, we will prove the uniqueness of the strong solutions of the problem (1). Let $(u, d)$ and $(\bar{u}, \bar{d})$ be the two solutions for the problem (1) with the same $u_{0}, d_{0}$. Assume that $(\hat{u}, \hat{d})=(\bar{u}-u, \bar{d}-d)$. Then we have

$$
\begin{aligned}
& \frac{1}{2} \frac{d}{d t}\left(\|\hat{u}\|_{L^{2}}^{2}+\|\nabla \hat{d}\|_{L^{2}}^{2}\right)+\|\nabla \hat{u}\|_{L^{2}}^{2}+\|\Delta \hat{d}\|_{L^{2}}^{2}+\alpha \int_{D}\left(|\bar{u}|^{\beta-1} \bar{u}-|u|^{\beta-1} u\right)(\bar{u}-u) d x \\
& \leq \int_{D}|\hat{u}|^{2}|\nabla \bar{u}| d x+\int_{D}|\hat{u}||\nabla \hat{d}||\Delta \bar{d}| d x \\
& \quad+\int_{D}|\bar{u}||\nabla \hat{d}||\Delta \hat{d}| d x+\int_{D}|f(\bar{d})-f(d)||\Delta \hat{d}| d x \\
& =\sum_{i=1}^{4} J_{i}(t) .
\end{aligned}
$$

Since $g(u)=\alpha|u|^{\beta-1} u$ is a monotonic function in $D$, it is easy to get

$$
\alpha \int_{D}\left(|\bar{u}|^{\beta-1} \bar{u}-|u|^{\beta-1} u\right)(\bar{u}-u) d x \geq 0
$$

For $J_{1}(t)$, using the Gagliardo-Nirenberg and Young inequalities, we have

$$
\begin{aligned}
J_{1}(t) & \leq C\|\hat{u}\|_{L^{2}}^{\frac{1}{2}}\|\nabla \hat{u}\|_{L^{2}}^{\frac{3}{2}}\|\nabla \bar{u}\|_{L^{2}} \\
& \leq \frac{1}{4}\|\nabla \hat{u}\|_{L^{2}}^{2}+C\|\nabla \bar{u}\|_{L^{2}}^{4}\|\hat{u}\|_{L^{2}}^{2} .
\end{aligned}
$$


For $J_{2}(t)$ and $J_{3}(t)$, similarly, we also get

$$
\begin{aligned}
J_{2}(t) & \leq C\|\hat{u}\|_{L^{4}}\|\nabla \hat{d}\|_{L^{4}}\|\Delta \bar{d}\|_{L^{2}} \\
& \leq C\|\hat{u}\|_{L^{2}}^{\frac{1}{4}}\|\nabla \hat{u}\|_{L^{2}}^{\frac{3}{4}}\|\nabla \hat{d}\|_{L^{2}}^{\frac{1}{4}}\|\Delta \hat{d}\|_{L^{2}}^{\frac{3}{4}}\|\Delta \bar{d}\|_{L^{2}} \\
& \leq \frac{1}{8}\|\nabla \hat{u}\|_{L^{2}}^{2}+\frac{1}{8}\|\Delta \hat{d}\|_{L^{2}}^{2}+C\|\Delta \bar{d}\|_{L^{2}}^{4}\left(\|\hat{u}\|_{L^{2}}^{2}+\|\nabla \hat{d}\|_{L^{2}}^{2}\right)
\end{aligned}
$$

and

$$
\begin{aligned}
J_{3}(t) & \leq C\|\bar{u}\|_{L^{6}}\|\nabla \hat{d}\|_{L^{2}}^{\frac{1}{2}}\|\Delta \hat{d}\|_{L^{2}}^{\frac{3}{2}} \\
& \leq \frac{1}{8}\|\Delta \hat{d}\|_{L^{2}}^{2}+C\|\nabla \bar{u}\|_{L^{2}}^{4}\|\nabla \hat{d}\|_{L^{2}}^{2} .
\end{aligned}
$$

For $J_{4}(t)$, applying the Hölder inequality, we get

$$
\begin{aligned}
J_{4}(t) & \leq\|f(\bar{d})-f(d)\|_{L^{2}}\|\Delta \hat{d}\|_{L^{2}} \\
& \leq \frac{1}{4}\|\Delta \hat{d}\|_{L^{2}}^{2}+C\left(1+\|\nabla \bar{d}\|_{L^{2}}^{4}+\|\nabla d\|_{L^{2}}^{4}\right)\|\nabla \hat{d}\|_{L^{2}}^{2} .
\end{aligned}
$$

Summing (16)-(21), we have

$$
\begin{aligned}
& \frac{d}{d t}\left(\|\hat{u}\|_{L^{2}}^{2}+\|\nabla \hat{d}\|_{L^{2}}^{2}\right)+\|\nabla \hat{u}\|_{L^{2}}^{2}+\|\Delta \hat{d}\|_{L^{2}}^{2} \\
& \quad \leq C\left(1+\|\nabla \bar{u}\|_{L^{2}}^{4}+\|\nabla \bar{d}\|_{L^{2}}^{4}+\|\nabla d\|_{L^{2}}^{4}+\|\Delta \bar{d}\|_{L^{2}}^{4}\right)\left(\|\hat{u}\|_{L^{2}}^{2}+\|\nabla \hat{d}\|_{L^{2}}^{2}\right) .
\end{aligned}
$$

Applying the Gronwall inequality and (7) and (15), then we have

$$
\|\hat{u}(t)\|_{L^{2}}^{2}+\|\nabla \hat{d}(t)\|_{L^{2}}^{2} \leq\left(\|\hat{u}(0)\|_{L^{2}}^{2}+\|\nabla \hat{d}(0)\|_{L^{2}}^{2}\right) e^{C \int_{0}^{t} H(s) d s}
$$

where, $H(s)=1+\|\nabla \bar{u}(s)\|_{L^{2}}^{4}+\|\nabla \bar{d}(s)\|_{L^{2}}^{4}+\|\nabla d(s)\|_{L^{2}}^{4}+\|\Delta \bar{d}(s)\|_{L^{2}}^{4}$. The uniqueness of the strong solutions of the problem (1) is proved. This completes the proof of Theorem 1.1.

\section{Acknowledgements}

The authors are thankful to the learned reviewers for their time and efforts in reviewing our article.

\section{Funding}

The first author is supported by the Natural Science Foundation of Shandong Province under Grant No. ZR2018QA002, the National Natural Science Foundation of China No. 11901342 and China Postdoctoral Science Foundation No. 2019M652350. The second author is supported by the NSF of China (No. 11701269). The third author is supported by the NSF of China (No. 11371183) and the NSF of Shandong Province (No. ZR2013AM004).

\section{Availability of data and materials}

Not applicable.

\section{Competing interests}

The authors declare that they have no competing interests regarding the publication of this paper.

\section{Authors' contributions}

The authors have equally contributed to the manuscript. All authors read and approved the final manuscript.

\section{Author details}

'School of Mathematical Sciences, Qufu Normal University, Qufu, P.R. China. ${ }^{2}$ School of Applied Mathematics, Nanjing University of Finance and Economics, Nanjing, P.R. China. ${ }^{3}$ School of Mathematics and Statistics, Ludong University, Yantai, P.R. China. 


\section{Publisher's Note}

Springer Nature remains neutral with regard to jurisdictional claims in published maps and institutional affiliations.

Received: 9 November 2019 Accepted: 15 April 2020 Published online: 29 April 2020

\section{References}

1. Lin, F.H.: Nonlinear theory of defects in nematic liquid crystals; phase transition and flow phenomena. Commun. Pure Appl. Math. 42, 789-814 (1989)

2. Lin, F.H., Liu, C.: Nonparabolic dissipative systems modeling the flow of liquid crystals. Commun. Pure Appl. Math. 48 501-537 (1995)

3. Hajduk, K.W., Robinson, J.C.: Energy equality for the 3D critical convective Brinkman-Forchheimer equations. J. Differ. Equ. 263, 7141-7161 (2017)

4. Liu, H., Gao, H.J.: Decay of solutions for the 3D Navier-Stokes equations with damping. Appl. Math. Lett. 68, 48-54 (2017)

5. Liu, H., Gao, H.J.: Ergodicity and dynamics for the stochastic 3D Navier-Stokes equations with damping. Commun. Math. Sci. 16, 97-122 (2018)

6. Markowich, P.A., Titi, E.S., Trabelsi, S.: Continuous data assimilation for the three-dimensional Brinkman-Forchheimer-extended Darcy model. Nonlinearity 29, 1292-1328 (2016)

7. Lin, F.H., Wang, C.Y.: Gobal existence of weak solutions of the nematic liquid crystal flow in dimension three. Commun. Pure Appl. Math. 69, 1532-1571 (2016)

8. Liu, H., Sun, C.F., Meng, F.W.: Global well-posedness of the 3D magneto-micropolar equations with damping. Appl. Math. Lett. 94, 38-43 (2019)

\section{Submit your manuscript to a SpringerOpen ${ }^{\odot}$ journal and benefit from:}

- Convenient online submission

Rigorous peer review

Open access: articles freely available online

High visibility within the field

- Retaining the copyright to your article 